\title{
A LABORATORY-BASED X-RAY PHASE CONTRAST IMAGING SCANNER WITH APPLICATIONS IN BIOMEDICAL AND NON-MEDICAL DISCIPLINES
}

\author{
C. K. HAGEN*, P. C. DIEMOZ*, M. ENDRIZZI*, P. R. T. MUNRO^, M. B. SZAFRANIEC*, \\ T. P. MILLARD*, R. SPELLER, D A. OLIVO* \\ *Department of Medical Physics and Bioengineering, University College London, London WC1E 6BT, UK \\ charlotte.hagen.10@ucl.ac.uk \\ $\wedge$ Optical + Biomedical Engineering Laboratory, School of Electrical, Electronic and Computer Engineering, \\ The University of Western Australia, Crawley, Western Australia 6009, Australia \\ ${ }^{\wedge}$ Centre for Microscopy, Characterisation and Analysis, The University of Western Australia, Crawley, \\ Western Australia 6009, Australia
}

Published 25 February 2014

\begin{abstract}
X-ray phase contrast imaging (XPCi) provides a much higher visibility of low-absorbing details than conventional, attenuation-based radiography. This is due to the fact that image contrast is determined by the unit decrement of the real part of the complex refractive index of an object rather than by its imaginary part (the absorption coefficient), which can be up to 1000 times larger for energies in the $\mathrm{X}$-ray regime. This finds applications in many areas, including medicine, biology, material testing, and homeland security. Until lately, XPCi has been restricted to synchrotron facilities due to its demanding coherence requirements on the radiation source. However, edge illumination XPCi, first developed by one of the authors at the ELETTRA Synchrotron in Italy, substantially relaxes these requirements and therefore provides options to overcome this problem. Our group has built a prototype scanner that adapts the edge-illumination concept to standard laboratory conditions and extends it to large fields of view. This is based on $\mathrm{X}$-ray sources and detectors available off the shelf, and its use has led to impressive results in mammography, cartilage imaging, testing of composite materials and security inspection. This article presents the method and the scanner prototype, and reviews its applications in selected biomedical and non-medical disciplines.
\end{abstract}

Keywords: X-ray imaging; phase contrast; phase retrieval.

\section{Introduction}

When X-rays traverse an object, they experience absorption and a phase shift that, if spatially non-uniform, translates into a slight deviation from their original path. This can be expressed by characterizing objects through their complex refractive index:

This is an Open Access article published by World Scientific Publishing Company. It is distributed under the terms of the Creative Commons Attribution 3.0 (CC-BY) License. Further distribution of this work is permitted, provided the original work is properly cited. 


$$
n=1-\delta+i \beta
$$

The imaginary part $\beta$ in Eq. (1) is directly proportional to the attenuation coefficient and drives the corresponding effect, while the decrement from unity of its real part $\delta$ (in the following referred to as refractive index decrement) is responsible for phase effects such as refraction. Within the energy range of X-rays used in biomedical applications, the refractive index decrement of an object can be up to three orders of magnitude larger than its absorption term. Hence, if $\delta$ is exploited for contrast generation rather than $\beta$, a significantly better visibility of low absorbing details can be expected.

Conventional radiographic techniques exploit the absorption properties of an object for image formation. X-ray phase contrast imaging (XPCi) techniques, conversely, are characterized by the fact that image contrast is not only due to variations of $\beta$ but also (or entirely) due to variations of $\delta$ within an object. Exploiting phase effects in order to obtain increased image contrast finds application where conventional (absorption-based) radiography is limited: i.e., when low absorbing details need to be visualized and when details with similar absorption properties need to be distinguished in an image. This occurs in various biomedical and non-medical disciplines such as mammography, material testing or homeland security.

The first XPCi techniques have been developed in the mid-nineties, mainly at synchrotrons, and impressive results have been published. ${ }^{1-3}$ Free space propagation ${ }^{2}$ (FSP) imaging can be considered the most straightforward XPCi method in terms of its implementation: a detector is placed at a distance from the imaged object instead of in contact with it. As a consequence, X-rays that have been phase shifted can interfere with non-phase shifted ones, and an interference pattern can be detected provided the detector has a sufficiently high spatial resolution. As for requirements on the spatial coherence, FSP is restricted to synchrotrons and micro-focal X-ray sources. Another approach is analyzer-based imaging $(\mathrm{ABi}){ }^{3}$ Here, a crystal is placed between the sample and the detector, reflecting and absorbing portions of the beam depending on the angle with which X-rays impinge on its surface. If the crystal is rocked slightly off the Bragg angle, its sensitivity to beam deviations caused by an object placed upstream of it is substantially increased: if the beam direction is changed such that it hits the crystal further off or closer to the Bragg angle, a decrease or an increase of intensity with respect to the background can be measured. ABi works best with a non-divergent monochromatic beam, conditions which are difficult to achieve outside synchrotron facilities due to limited X-ray flux.

More recently, the focus of research has been on developing methods that can make XPCi compatible with conventional X-ray sources and detectors, as this is the first step towards a transfer into laboratory environments and eventually into clinical practice. One such approach is Talbot ("grating") interferometry, ${ }^{4}$ a technique making use of the Talbot self-imaging effect. A phase grating generates an interference pattern that is a replica of the grating pattern itself at a given set of (Talbot) distances; typically an absorption grating is placed at one of these distances, with a detector immediately behind it. An object introduced upstream of the phase grating distorts the interference pattern. The 
distortion can be quantified by scanning one of the gratings in the transverse (i.e., orthogonal both to the grating trenches and X-ray propagation) direction and by comparing the distorted intensity with the non-distorted one. The method relies on a coherent source, as the formation of a Talbot self-image is an intrinsically coherent effect. However, the method works under relaxed coherence conditions if a third grating is placed in front of the source, ${ }^{5}$ generating individually coherent but mutually incoherent source-lets. Adding this source grating corresponds to switching from a Talbot to a Talbot/Lau configuration. Although impressive results have been obtained with this technique, the method has several limitations. First, Talbot distances are energy dependent, which causes a problem if a polychromatic source is employed. Second, the period of the employed gratings is of the order of a few microns, which makes the system sensitive to environmental vibrations and difficult to scale up to large fields of view. ${ }^{6}$

This article describes a different XPCi method that is compatible with conventional $\mathrm{X}$-ray equipment. It originates from synchrotron experiments carried out at the SYRMEP (synchrotron radiation for medical physics) beamline at ELETTRA (Trieste, Italy) in the late nineties, in which a vertically narrow beam was aligned with the edge of a single row of detector pixels rather than with the pixel center (see Fig. 1a)). This edge illumination (EI) principle ${ }^{7}$ results in sensitivity to deviations of the narrow beam when an object is introduced: if the beam is deviated out of the pixel, less X-rays are detected with respect to the background, and vice versa, if the beam is deviated into the pixel, more X-rays are detected. By scanning an object through the narrow beam, an image is built up. By means of opportunely designed X-ray masks, this setup can be extended to large fields of view, and the need to scan the object can be overcome by replicating the EI configuration for multiple rows of pixels. Two sets of masks, sometimes termed coded apertures (CA), are required. ${ }^{8-9}$ One set of CAs is placed immediately in front of the detector and creates insensitive regions between the pixels (detector mask). The other set is positioned immediately upstream of the object, where it splits the incoming beam into individual beamlets (sample mask). If the two sets of apertures are aligned with respect to each other in such a way that each of the beamlets hits the edge of one of the apertures in the detector mask, the EI principle is simultaneously achieved for each row of pixels in a $2 \mathrm{D}$ area detector.

The EI principle has recently been demonstrated to be extremely sensitive to phase effects when implemented at synchrotrons: notably, refraction angles on the nanoradian scale can be resolved. ${ }^{10}$ Besides being a valuable imaging tool at synchrotrons, EI/CA XPCi can be implemented in laboratory environments ${ }^{9}$ as it works with X-ray sources with focal spots of up to $100 \mu \mathrm{m}$, compatible for example with the state of the art of clinical mammography sources. The beam polychromaticity is not a problem either as the signal is a weighted sum of the signals at the energies of the spectrum; no selection of a narrow energy band takes place; hence the X-ray flux is used efficiently. The use of commercially available detectors is straightforward, the only limiting factor being excessively high cross-talk between pixels, as this has been shown to decrease image contrast; ${ }^{11}$ however, detectors with significant cross-talk can be used in combination with 


\section{K. Hagen et al.}

"line skipping" CA masks. The masks can be manufactured up to large fields of view as no nanofabrication is necessary (their period matches the detector pixel size, typically 50-100 $\mu \mathrm{m})$. Based on the above concepts, a CA XPCi scanner prototype has been built and is fully operational in the radiation physics laboratories at UCL.

\section{Materials and Methods}

A sketch of an EI setup for a single pixel row and a full area detector (CA setup) can be seen in Fig. 1. While at synchrotrons, the source-to-sample distance is typically very large with respect to the sample-to-detector distance, compact laboratory setups with source-to-detector distances of under two meters are possible. The period of the coded apertures is designed to match the detector pixel size; however, the period of the sample apertures needs to be downscaled in order to account for the magnification in the case of a divergent beam. The aperture size is a fraction (typically $<0.5$ ) of the period. It should be noted that EI and CA XPCi are totally non-interferometric methods. In addition to the used source being incoherent, the beamlets in the CA setup are separated by a distance sufficiently large for them not to interfere with each other - typically they do not even overlap.

a)

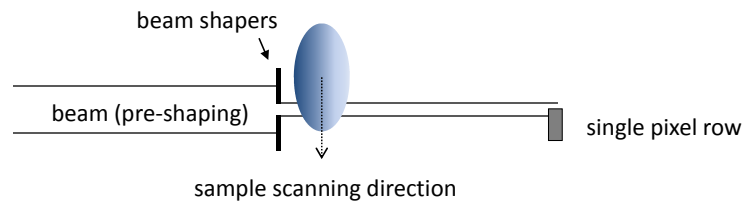

b)

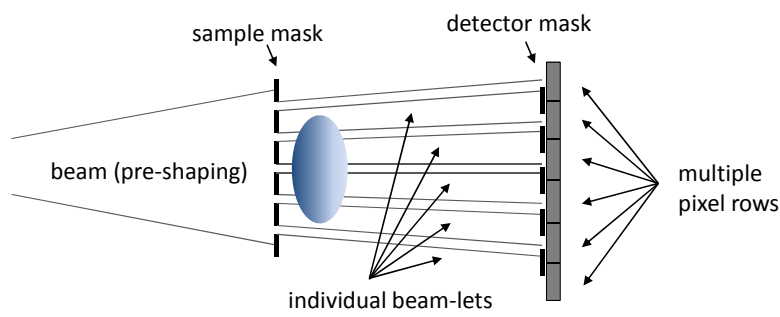

Fig. 1. Schematic of an edge illumination setup using a single pixel row (a) and its coded aperture extension to a full 2D detector (b).

If the detector mask is kept still and the sample mask scanned in the direction transverse to the apertures, a periodic intensity pattern can be measured. This "illumination curve" assumes its minimum value when the absorbing parts of the sample mask cover the transmitting parts of the detector mask and its maximum value when the transmitting parts of both sets of apertures overlap. Positioning the sample apertures such that the beamlets hit the edges of the insensitive regions created by the detector apertures thus corresponds to the slopes of the illumination curve. If the position is changed from 
one slope to the other, the refraction signal in the image is inverted: beamlets that had previously been deviated out of the apertures in the detector mask and created an intensity loss are now deviated into it, causing a gain in intensity. A CA setup as described above is sensitive to phase effects only in one dimension (perpendicular to the mask apertures, which are effectively long slits). However, the phase sensitivity can easily be extended to two dimensions by using L-shaped apertures. ${ }^{12}$

The spatial resolution in a CA XPCi setup is not determined by the pixel size but rather by the size of the individual beamlets, i.e., the apertures in the sample mask. However it should be noted that intrinsically, the beamlets do not illuminate the entirety of the object, hence some degree of object information (albeit at the sub-pixel level) may be missing in the image. This can be overcome by taking multiple images while the object is being displaced by sub-pixel amounts; these images are then combined in a single image by altering columns taken from the individual images ("dithering"). A dithered image can reveal features that are much smaller than the pixel size.

The EI method and its CA extension have been thoroughly investigated over the recent years. Two mathematical models have been presented and experimentally validated: one based on geometrical optics" ("ray tracing") and one based on wave optics. ${ }^{13}$ The wave optical model has been shown to take coherence effects into account, if present, and to converge to the ray tracing one under relaxed coherence conditions. More recently, we demonstrated that quantitative EI and CA XPCi are possible. A dedicated phase retrieval algorithm ${ }^{14}$ has been developed to separate and quantify the phase and the absorption contributions that are typically present in an image. All it requires is knowledge of the illumination curve and two images acquired on two opposite slopes (which corresponds to illuminating the two opposite edges of each aperture in the detector mask). The extracted phase contribution is equal to the angle of refraction introduced by an object:

$$
\Delta v=\frac{1}{k} \partial / \partial x \int_{O b j} \delta d z
$$

In practice, the algorithm in Eq. (2) establishes a line integral relationship between the measured data and the refractive index decrement of the object, which implies the feasibility of extending EI and CA XPCi to a tomographic imaging modality.

The CA XPCi scanner prototype built and in operation at UCL comprises the following equipment. A Rigaku M007 X-ray source (Rigaku Corporation, Tokyo, Japan) with a rotating molybdenum target is used. The source focal spot has been measured to be $70 \mu \mathrm{m}$ in the horizontal direction (the direction of phase sensitivity). The source is typically operated at $35 \mathrm{kV}$ and $25 \mathrm{~mA}$. As a detector, we use the Anrad "SMAM" amorphous selenium direct conversion flat panel (Analogic Canada Corporation, SaintLaurent, Quebec, Canada) with a pixel size of $85 \mu \mathrm{m}^{2}$. The aperture masks have been manufactured to the authors' design by Creatv Microtech Inc. (Potomac, MD), by electroplating a gold layer on a graphite substrate. Both the sample and the detector masks are mounted on Newport translation and rotation stages (Newport Corporation, Irvine, CA) and Kohzu goniometers (Kohzu Precision Co., Ltd., Kawasaki Kanagawa, 


\section{K. Hagen et al.}

Japan), allowing for fine alignment with all degrees of freedom. The source-to-sample distance is $1.6 \mathrm{~m}$, and the sample-to-detector distance is $0.4 \mathrm{~m}$. The apertures in the prototype currently cover a field of view of $5 \mathrm{~cm}^{2}$ and are obtained in a $30 \mu \mathrm{m}$ thick gold layer. The pitch of the detector apertures is $83.5 \mu \mathrm{m}$ with a $24 \%$ open fraction, and the pitch sample aperture is $66.8 \mu \mathrm{m}$ with an $18 \%$ open fraction. A graphical 3D rendering of the scanner prototype can be seen in Fig. 2.

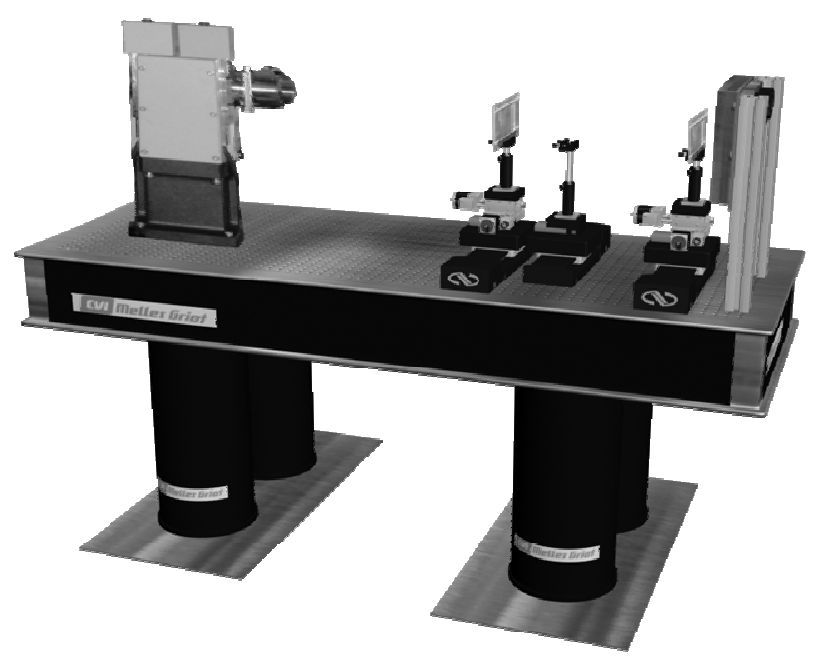

Fig. 2. Volume graphical rendering of the XPCi scanner prototype in operation in the radiation physics laboratories at UCL.

\section{Results and Discussion}

Figure 3 shows images of a geranium flower acquired with the UCL scanner prototype. Figures $3 a$ ) and $3 b$ ) show the images acquired on the two opposite slopes of the illumination curve, while Figs. 3c) and 3d) show the extracted refraction and absorption images, respectively. The experimental conditions were the following: source operated at $35 \mathrm{kV}$ and $25 \mathrm{~mA}$, no beam filtration, and sample apertures shifted by $\pm 8 \mu \mathrm{m}$ with respect to the "maximum" position to acquire the images on the left and the right slope of the illumination curve. The object has been dithered with eight steps $(8.4 \mu \mathrm{m}$ each) in order to increase the spatial resolution. Five frames have been acquired and averaged at each dithering step, and the exposure time for each frame was $7 \mathrm{~s}$. Images a) and b) have been corrected for gain and offset of the detector before being input to the phase retrieval algorithm yielding images c) and d).

Recently our group has investigated applications within biomedical and non-medical disciplines that can benefit from CA XPCi. A recent publication ${ }^{15}$ has demonstrated that the CA XPCi scanner can correctly visualize thin cartilage layers, a notoriously challenging sample for X-rays. A knee joint dissected from a rat has been imaged both in air and in a saline solution to mimic in-vivo conditions, and in both cases the $100 \mu \mathrm{m}$ 
cartilage layer was correctly visualized. These results can have a substantial impact on research on the causes of osteoarthritis, a disease whose origin is still not fully understood and for which currently there is no cure.
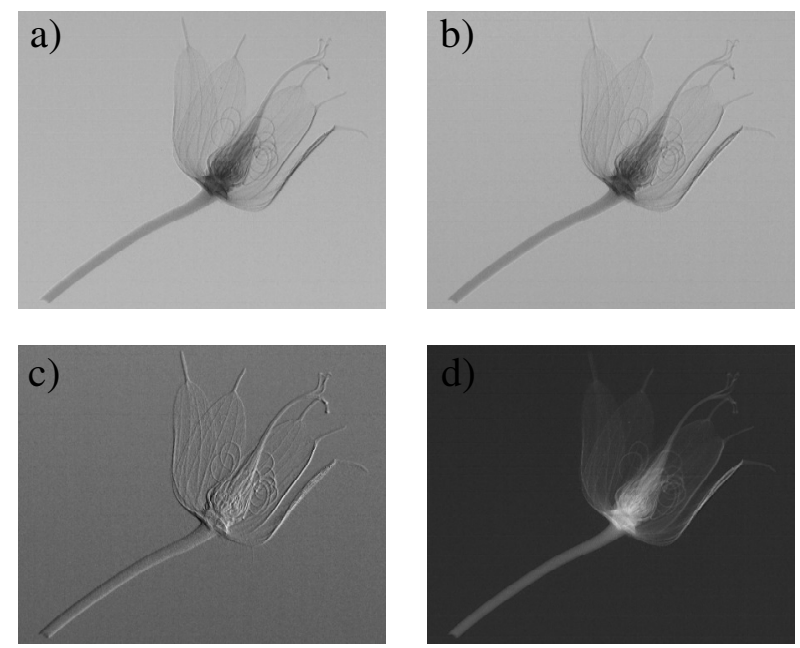

Fig. 3. Images of a geranium flower acquired with the UCL phase contrast scanner: a) and b) are the mixed phase and absorption contrast images acquired at the left and right slopes of the illumination curve, c) shows the extracted refraction angle image and d) shows the extracted absorption image.

An example of a non-medical application of CA XPCi has been proposed in homeland security. While the bulky elements of threat objects such as bombs can be easily spotted by a conventional system, these can also be confused with other objects found in suitcases thus giving rise to false alarms. On the other hand, the detection of fine detonator components (essential in identifying the object as a threat), such as thin wires, can be very challenging for a conventional security scanner. Images of a custom-made "bomb" phantom consisting of a bulky plastic object (the "explosive") with thin metallic wires sticking out of it have been taken with the CA XPCi scanner and with a conventional radiographic method. The CA XPCi images have correctly revealed the wires, which remained invisible in the conventional image. ${ }^{16}$ This is just an example of non-medical applications of CA XPCi, and others could be mentioned, such as for example, the recent demonstration of the detection of faint blemishes in composite materials invisible to conventional methods. ${ }^{17}$

\section{Conclusions}

We have presented a totally incoherent XPCi method that is based on the edge illumination principle. The proposed method provides enhanced sensitivity at synchrotrons and is easily adapted to laboratory based setups. A prototype scanner has been built and successfully tested on a number of significant applications in both medical and non-medical disciplines. 


\section{Acknowledgements}

This work is funded by the UK Engineering and Physical Sciences Research Council (Grants EP/G004250/1 and EP/I021884/1).

\section{References}

1. S. W. Wilkins, T. E. Gureyev, D. Gao, A. Pogany and A. W. Stevenson, Nature 384, 335 (1996).

2. A. Snigirev, I. Snigireva, V. Kohn, S. Kuznetsov and I. Schelokov, Rev. Sci. Instrum. 66, 5486 (1995).

3. V. N. Ingal and E. A. Beliaevskaya, J. Phys. D: Appl. Phys 28, 2314 (1995).

4. C. David, B. Nöhammer, H. H. Solak and E. Ziegler, Appl. Phys. Lett. 81, 3287 (2002).

5. F. Pfeiffer, T. Weitkamp, O. Bunk and C. David, Nat. Phys. 2, 258 (2006).

6. T. Weitkamp, A. Diaz, C. David, F. Pfeiffer, M. Stampanoni, P. Cloetens and E. Ziegler, Optics Express 13, 6296 (2005).

7. A. Olivo, F. Arfelli, G. Cantatore, R. Longo, R. H. Menk, S. Pani, M. Prest, P. Poropat, L. Rigon, G. Tromba, E. Vallazza and E. Castelli, Med. Phys. 28, 1610 (2001).

8. A. Olivo and R. Speller, Appl. Phys. Lett. 91, 074106 (2007).

9. A. Olivo and R. Speller, Phys. Med. Biol. 52, 6555 (2007).

10. P. C. Diemoz, M. Endrizzi, C. E. Zapata, Z. D. Pešić, C. Rau, A. Bravin, I. K. Robinson and A. Olivo, Phys. Rev. Lett. 110, 138105 (2013).

11. K. Ignatyev, P. R. T. Munro, R. D. Speller and A. Olivo, Rev. Sci. Instrum. 82, 073702 (2011).

12. A. Olivo, S. E. Bohndiek, J. A. Griffiths, A. Konstantinidis and R. D. Speller, Appl. Phys. Lett. 94, 044108 (2009).

13. P. R. Munro, K. Ignatyev, R. Speller and A. Olivo, Optics Express 18, 4103 (2010)

14. P. R. Munro, C. K. Hagen, M. B. Szafraniec and A. Olivo, Optics Express 21, 11187 (2013).

15. M. Marenzana, C. K. Hagen, P. Das Neves-Borges, M. Endrizzi, M. B. Szafraniec, K. Ignatyev and A. Olivo, Phys. Med. Biol. 57, 8173 (2012).

16. A. Olivo, K. Ignatyev, P. R. Munro and R. Speller, Appl. Opt. 50, 1765 (2011).

17. M. Endrizzi, P. C. Diemoz, M. B. Szafraniec, C. K. Hagen, T. P. Millard, C. E. Zapata, P. R. T. Munro, K. Ignatyev, M. Marenzana, R. D. Speller and A. Olivo, Proc. SPIE 8668, 866812 (2013). 